\title{
Risco positivo na atividade de crédito que otimiza o desempenho econômico- financeiro de cooperativas de crédito*
}

\author{
Positive risk in credit activity that optimizes the financial performance of credit \\ unions
}

\section{Risque positif de l'activité de crédit qui optimise la performance financière des caisses de crédit}

\author{
Riesgo positivo de la actividad crediticia, que optimiza el rendimiento financiero de las \\ cooperativas de crédito
}

\author{
Tarcísio Pedro da Silva** \\ (tarcisio@furb.br) \\ Nelson Hein** \\ (hein@furb.br)
}

Recebido em 08/07/2015; revisado aprovado em 14/07/2015; aceito em 20/07/2015.

DOI: http://dx.doi.org/10.20435/1984042X2016213

\begin{abstract}
Resumo: A pesquisa objetiva identificar o nível de risco positivo na atividade de crédito que otimiza o desempenho econômico-financeiro de cooperativas de crédito. A Teoria de Finanças consubstancia a pesquisa, no desempenho econômico-financeiro e na determinação do risco positivo em pesquisa, nas dez maiores cooperativas de crédito brasileiras. $\mathrm{O}$ desempenho econômico-financeiro permitiu a validação do grau de risco positivo no desempenho da atividade de crédito, que se apresenta de forma conservadora e equalizada nas cooperativas.

Palavras-chaves: risco positivo; desempenho econômico-financeiro; cooperativas de crédito.

Abstract: The research aims to identify the positive level of risk in the credit activity that optimizes the financial performance of credit unions. Finance Theory substantiates the research, financial performance and in determining the positive risk research in the ten largest Brazilian credit unions. The financial performance has enabled the positive risk level validation on the performance of credit activity, which appears conservative and equalized in form cooperatives.
\end{abstract}

Key words: positive risk; financial performance; credit unions.

Résumé: La recherche vise à identifier le niveau de risque positif dans l'activité de crédit qui optimise la performance financière des caisses de crédit. Théorie des Finances justifie la recherche, la performance financière et dans la détermination de la recherche sur les risques positifs dans les dix plus grandes coopératives de crédit brésiliennes. La performance financière a permis la validation du niveau de risque positif sur la performance de l'activité de crédit, qui semble conservateur et égalisé en forme des coopératives.

Mots-clés: risque positif; performance économique et financière; coopératives de crédit.

Resumen: La investigación tiene como objetivo identificar el nivel de riesgo positivo en la actividad de crédito que optimiza el rendimiento financiero de las cooperativas de crédito. Finanzas Teoría corrobora la investigación, el rendimiento financiero y en la determinación de la investigación de riesgo positivo en los diez cooperativas más grandes de Brasil. El rendimiento financiero ha permitido la validación positiva nivel de riesgo en el desarrollo de la actividad de crédito, que aparece conservador y ecualizado en forma de cooperativas.

Palabras clave: riesgo positivo; desempeño financeiro; las cooperativas de crédito.

\section{INTRODUÇÃO}

As atividades iniciais que envolvem aspectos motivacionais em prol do crédito foram relacionadas ao crédito agrícola, que data do século XV. Presentemente, o número de cooperativas de crédito é superior a 1370, com atendimento a mais de cinco milhões de associados (BACEN, 2010). A característica das cooperativas de crédito está no trata- mento dos cooperados como sendo sócios. Dessa forma, havendo aumento no resultado financeiro da cooperativa, os sócios recebem da parte destinada a distribuição das sobras ou o aumento da cota capital.

As análises de Meehling (1959) destacam que, na época, as cooperativas estavam chegando aos cinquenta anos de cooperativas de crédito e já mostraram um crescimento rápido, nas duas décadas anteriores, atraíram

\footnotetext{
* Artigo apresentado no Congresso Brasileiro de Custos, 2013, Uberlândia, MG.

** Universidade Regional de Blumenau (FURB), Blumenau, Santa Catarina, Brasil.
} 
a atenção da comunidade bancária, que tem consciência do fenomenal crescimento dessas sociedades e tende a vê-los como concorrentes.

Moody e Fite (1971) destacaram que não se tem registros certos da origem das cooperativas de crédito. Porém destacam indícios pela atividade agrícola, de artesãos, pequenos produtores que, sempre na tentativa de melhorar sua posição na sociedade capitalista, em ambiente moderno e não feudal, buscavam também a restauração do espírito comunitário.

$\mathrm{Na}$ atividade de crédito, que envolve o gerenciamento de risco de crédito, Duffie e Singleton (2003) destacam que, além de considerar as dimensões estratégicas mais amplas de gerenciamento de riscos, os gestores seniores das instituições financeiras atuam, também, no desenvolvimento de políticas de crédito e no desenvolvimento de sistemas que permitam o gerenciamento da atividade.

$\mathrm{Na}$ mensuração do desempenho das atividades financeiras, proposta por Meyer e Pifer (1970), os quais estabelecem nove indicadores de previsão de falência dos bancos, os autores destacaram que, em uma economia de pleno emprego, a falência de uma empresa é a primeira prova da má utilização dos recursos.

A pesquisa de Sinkey Jr. (1975) ressaltou o trabalho iniciado por Meyer e Pifer (1970), analisou os problemas dos bancos, com destaque para bons indicadores discriminantes, tais como a composição de ativos, as características do empréstimo, a adequação de capital, as origens e as aplicações das receitas, a eficiência e a rentabilidade. Stuhr e Wicklen (1974) destacam a utilização de dados publicados pelos bancos para análise da situação econômico-financeira, com ênfase para a estruturação apresentada pelo modelo CAMEL que considera os cinco grupos de indicadores (Capital adequacy, Asset quality, Management expertise, Liquidity and Sensitivity to market risk), que expressam adequação do capital, qualidade dos ativos, capacidade de gerenciamento, eficiência dos retornos, liquidez e sensibilidade ao risco de mercado.

O crescimento natural, em decorrência das atividades de crédito, na forma como se desenvolvem as cooperativas, obterá uma evolução natural, ao passo que, com a adoção de mecanismos propulsores no desempenho econômico-financeiro (HULME, 2000), pode resultar um desempenho modificado.

Dadas às situações expostas, surge a lacuna de pesquisa em direção à identificação, dentre outros aspectos, da otimização do risco positivo na atividade econômico-financeira das cooperativas de crédito brasileiras, em que se destaca como questão de pesquisa, o seguinte questionamento: Qual o nível de risco positivo na atividade de crédito que otimiza o desempenho econômico-financeiro de cooperativas de crédito? O objetivo geral do estudo é identificar o nível de risco positivo na atividade de crédito que otimiza o desempenho econômico-financeiro de cooperativas de crédito.

As justificativas da pesquisa destacam Horrigan (1966) que aponta como úteis, para a determinação da classificação de participação corporativa, os indicadores financeiros e os dados contábeis. Ao analisar alguns indicadores facilmente disponíveis, pode-se aferir correspondência para empresas "falidas" versus "não falidas", em que vários índices financeiros foram bons preceptores de falência, enquanto outros, provavelmente mais amplamente usado, apresentaram prognósticos simplistas (BEAVER, 1966).

$\mathrm{Na}$ análise de crédito pelas empresas, a análise das demonstrações financeiras é apenas uma parte da avaliação do processo inteiro, mas é uma ligação muito importante. Figura como um dispositivo rápido e eficiente para detecção de riscos de crédito desfavoráveis e podem contribuir para que o gestor tome decisões com potencial desastroso (ALTMAN, 1968).

A pesquisa de Renwick (1969) utilizou dados históricos publicados nas demonstrações de resultado e no balanço patrimonial, num período de onze anos, de 1957 até 1967 inclusive, e destacou a possibilidade de analisar o comportamento dos investidores no gerenciamento de ativos. Considerando o período base para a pesquisa, de dez anos, o amparo está em Altman (1969), que analisou o potencial de falência corporativa, os retornos ao acionista e a avaliação de compartilhamento em que analisou os bancos após dez anos da sua falência.

Em relação aos modelos descritos na literatura, a escolha pelo modelo CAMEL (Capital adequacy, Asset quality, Management expertise, Liquidity and Sensitivity to market risk) se 
destaca em função de ser originário de pesquisas, conforme Meyer e Piffer (1970), Altman (1971), Stuhr e Wicklen (1974), Martin (1977), Avery, Belton e Goldberg (1988), Parliament, Lennan e Fulton (1990), Yeh (1996), Hunjak e Jakovčević (2001), Godlewsk (2003), que estruturaram o modelo de avaliação de desempenho econômico-financeiro em instituições financeiras; além de ser o modelo pioneiro destacado por pesquisas estrangeiras, foi o que apresentou extenso volume de pesquisas em periódicos de finanças.

O período de análise utilizado na pesquisa, de 2001 até 2010, se ampara na pesquisa de Aharony, Jones e Swary (1980), que se utilizaram de dados de dez anos para análise de risco e retorno das características de falência corporativa usando dados do mercado de capitais. Demirgüç-Kunt e Maksimovic (1996) analisaram empiricamente os efeitos do mercado de ações, especialmente o desenvolvimento de opções de financiamento das empresas usando dados de trinta países em desenvolvimento de países industriais no período de 1980 a 1991.

As variáveis, eficiência dos ativos, solvência, eficiência, tamanho (depósitos totais), estrutura organizacional e taxa de empréstimo sobre o ativo, conforme Stuhr e Wiclen (1974), permitem a classificação dos bancos, como 1 para bancos fortes (solventes) e até 4 para aqueles que dependem de uma análise da qualidade dos ativos do banco e que foram considerados fracos. Os índices financeiros são frequentemente usados para medir a solidez financeira global de um banco e a qualidade do seu gerenciamento (DZEAWUNI; TANKO, 2008).

O modelo de Verhulst (1845) preconiza que o período de projeção do crescimento populacional seja estabelecido como de longo prazo, em que, por se tratar de situações com impacto no ambiente macroeconômico, foi estabelecido o período de 15 anos para projeção da população economicamente ativa, pelo crescimento populacional, conforme Miranda e Lima (2010), pela política de energia, segundo Meng e Niu (2011).

Assim se justifica a realização desta pesquisa devido ao crescimento da população economicamente ativa, com utilização de indicadores de desempenho econômico-financeiros pelo risco positivo otimizado em cooperativas de crédito, conforme o aporte teórico elencado.

\section{RISCO POSITIVO E DESEMPPENHO ECONÔMICO-FINANCEIRO EM COOPERATIVAS}

A gestão de risco considerada por Barger (1938) se relaciona com a causa do risco, com base em políticas previamente estabelecidas, que pode seguir para maior ou menor aceitação pelo risco, com influência no seu grau. Considera-se que a gestão de risco sofre influências do ambiente externo, que foge do controle organizacional, mas com possibilidade de monitoramento.

Os destaques de Kaldor (1939) envolvem a oferta e procura no mercado, em que o recebimento posterior implica a remuneração adicional, porém não representa um ganho, mas uma remuneração adicional. Com isto, pode ocorrer a especulação no mercado em relação à venda da produção, com possibilidade do comportamento positivo ou negativo.

Conjuntamente, Dow (1940) esclareceu que o risco negativo pode ocorrer no caso especulativo em que o vendedor não dispõe do produto, o que destaca como risco negativo. Em relação ao risco positivo, que foge da especulação, destaca uma venda emparelhada, em que ocorre a compra e posteriormente a venda, como na compra de títulos.

A pesquisa de Hahn (1955) explicou que o risco positivo representa um valor superior aos preços esperados, que deve ser tratado de forma separada do custo. Dessa forma, ao apurar o risco positivo, faz-se necessária a exclusão do risco natural, que representa o momento anterior ao risco positivo. Com relação ao grau de risco, Avio (1974) explica que, na avaliação de alto risco, este se relaciona com os limites das taxas de juro. A escolha pelo alto grau de risco se relaciona com o esperado pelo retorno.

Cooley (1977) esclarece que a gestão do grau de risco implica a probabilidade de o resultado esperado ser diferente do real, a qual deve ser observada na relação investimento e retorno. Wolken e Navratil (1981) alertam que as taxas máximas de juros poderão afetar a competitividade da cooperativa de crédito na captação de depósitos. Com relação ao comportamento dos investidores, Grinblatt e 
Titman (1993) enfatizam que este pode apresentar uma forma agressiva nas opções de risco no investimento e resultar em retornos elevados, contudo se faz necessário apresentar persistência na busca pelo resultado, que pode sofrer interferência pelo tempo de espera no desempenho do investimento.

Já Marshall (2002, p. 36) enaltece que o fato de risco ser visto como algo negativo negligencia as opções positivas dele na "visão de risco como oportunidade. Gerenciar fatores positivos de risco significa avaliar as oportunidades existentes na assunção de risco. Os negócios são inerentemente arriscados; o sucesso chega àqueles que assumem riscos que dão certo". Andersen (2011) acrescenta que o crescimento das organizações, baseadas no conhecimento, pode reduzir o risco de queda e aumentar o potencial de ganho.

O risco positivo, neste trabalho, é representado pela taxa de crescimento na atividade de crédito, dado aumento da população economicamente ativa, que mantém o crescimento das cooperativas, como forma de alinhar os recursos disponíveis às oportunidades na gestão do crédito, sem a apresentação de situação de turbulência. Respeita-se o intervalo de risco positivo que poderá ser utilizado para estabelecimento do indicador de crescimento, nas atividades de crédito para as cooperativas de crédito.

Em relação ao desempenho econômico-financeiro em cooperativas, Meehling (1959) destacou que, em pesquisa realizada para análise das cooperativas de crédito, esta foi centrada nas operações como refletido nos dados de balanço. Equipara-se assim, à análise feita pela National Bureau of Economic Research, em seus estudos de lidar com as instituições financeiras em que se estendiam empréstimos de dinheiro parcelado para os consumidores.

A pesquisa de Helmberger e Hoos (1962) apontou a existência da reciprocidade quando os membros de uma cooperativa atuam na tentativa evolutiva dos resultados pretendidos, que, por conseguinte, retornam aos membros como forma de remuneração pelo serviço ou produto consumido.

As contribuições de Pettway e Sinkey Jr (1980) apresentam como sugestão que, ao analisar o desempenho de bancos, deve-se utilizar uma metodologia que agrupe resultados com possibilidade de alerta sobre o de- sempenho da instituição. Além disso, Secrist (1936) sugeriu que dados de contabilidade dos bancos devem ser usados para distinguir o desempenho insalubre no sistema bancário. Ainda, Pettway eSinkey Jr. (1980) informaram que, no entanto, só recentemente se tentou fazer uso mais eficiente desses dados no formulário de sistemas de alerta ou de vigilância. Esses sistemas podem ser usados para ajudar no agendamento de avaliação bancária e alocando mais eficientemente os recursos bancários, que também eram escassos.

Parliament, Lerman e Fulton (1990) destacaram que os medidores de desempenho financeiro das cooperativas precisam abordar cinco índices financeiros que destacam os objetivos corporativos e, portanto, pode ser esperada a revelação das diferenças entre as cooperativas e demais instituições financeiras. Os medidores são relacionados às medidas de rentabilidade, alavancagem, solvência, liquidez e eficiência.

\section{PROCEDIMENTOS DA PESQUISA}

Esta pesquisa apresenta sua caracterização, como descritiva, e busca aporte teórico na orientação de Hair Jr. et al. (2009, p. 85), em que "[...] tem seus planos estruturados e especificamente criados para medir as características descritas em uma questão de pesquisa". A busca documental, segundo Gil (1999, p. 45), "baseia-se em materiais que ainda não receberam um tratamento analítico ou que podem ser reelaborados de acordo com os objetivos da pesquisa". Os dados coletados foram extraídos do balanço patrimonial, da demonstração do resultado de sobras/ prejuízo, do relatório de administração e dos estatutos sociais. As demonstrações, o relatório de administração e o estatuto social destacam variáveis ainda não analisadas sob o ponto de vista da pesquisa.

Creswell (2010, p. 26-27) considera que "a pesquisa quantitativa é um meio para testar teorias objetivas, examinando a relação entre as variáveis. Tais variáveis, por sua vez, podem ser medidas tipicamente por instrumentos, para que os dados numéricos possam ser analisados por procedimentos estatísticos".

Para a população e amostra, foi destacado que, no Brasil, no final do ano de 2010, 
as cooperativas de crédito totalizavam 1.370 , com mais de 4.500 postos de atendimento ao cooperado e com mais de cinco milhões de cooperados (BACEN, 2011, p. 114). Ainda, em 2010, o BACEN publicou o ranking das cem maiores cooperativas do Brasil, com base em dezembro de 2010. Em relação à amostra, objeto de estudo desta pesquisa, foram selecionadas, segundo o ranking estabelecido pelo BACEN, as dez maiores cooperativas brasileiras, com período de análise de dez anos, de 2001 a 2010. Para esta pesquisa, foi considerada uma amostra intencional em "que podem ser escolhidos os casos a serem incluídos e, assim, chegar a amostras que sejam satisfatórias para as necessidades" (MATTAR, 1997, p, 270). Martins e Theóphilo (2007, p. 119) esclarecem que a amostra intencional, “De acordo com determinado critério é escolhido intencionalmente um grupo de elementos que irão compor a amostra".

Para delineamento da amostra, foi considerada a primeira variável utilizada pelo BACEN para estabelecer o ranking, os ativos totais, pois estes representam 16,68\% do total de ativos de todas as (1.370) cooperativas de crédito brasileiras e, 29,35\% do total de ativos das 100 maiores cooperativas. Do ranking geral, seguindo a classificação do BACEN, podem-se elencar as Cooperativas de Crédito conforme o quadro 1, Ranking das cooperativas de crédito brasileiras utilizadas na amostra, considerando os dados de dezembro de 2010.

\begin{tabular}{|c|l|c|}
\hline Ordem & \multicolumn{1}{|c|}{ Cooperativas } & Localização \\
\hline 1 & Credicitrus & Bebedouro/SP \\
\hline 2 & Cooperforte & Brasília/DF \\
\hline 3 & Cocred & Sertãozinho/SP \\
\hline 4 & Viacredi & Blumenau/SC \\
\hline 5 & Pioneira RS & Nova Petrópolis/RS \\
\hline 8 & Região dos Vales & Guariba/SP \\
\hline 9 & Coopecredi & Campo Mourão/PR \\
\hline 10 & Credicoamo & Medianeira/PR \\
\hline 11 & Cataratas do Iguaçu & Londrina/PR \\
\hline 12 & Norte do Paraná - Uniprime & . \\
\hline
\end{tabular}

Quadro 1 - Ranking das cooperativas de crédito brasileiras utilizadas na amostra Fonte: Portal do Cooperativismo de Crédito (2011).

Nesse ranking, considerando que o BACEN utilizou como base o ano de 2010, diferente do período de coleta dos dados desta pesquisa, 2011, foram substituídas as cooperativas de número 6 e 7:a) a União RS de Santa Rosa em que ocorreu a fusão de três cooperativas, no ano de 2010, destas a Serro Azul, a Grande Santa Rosa e a Missões; b) Processo idêntico ocorreu em 01/07/2009, com a União PR de Maringá, com o processo de fusão da Maringá PR, a Vale do Bandeirante PR e a Norte do Paraná PR. Essas fusões impossibili- taram a utilização dos dados das cooperativas de 2001 a 2010 necessários à pesquisa.

Em virtude das limitações, pelos períodos necessários, foram acrescidas as cooperativas que figuram, no ranking do BACEN, em décimo primeiro lugar, a Cataratas do Iguaçu, e, em décimo segundo lugar, a Norte do Paraná, completando a amostra intencional.

Considerada a formação do modelo CAMEL, as equações destacadas na análise seguem considerando os seguintes indicadores:

$$
\text { EQTA }=\frac{\text { Patrimônio Líquidc }}{\text { Ativo Total }}
$$

Em que o EQTA = Patrimônio Líquido sobre o Ativo Total.

$$
\text { EQTL }=\frac{\text { Patrimônio Líquido }}{\text { Empréstimo Total }}
$$

Que se utiliza como EQTL = Patrimônio Líquido sobre Empréstimo Total. 


$$
\text { NPLTA }=\frac{\text { Empréstimo não Recebidc }}{\text { Ativo Total }}
$$

Para o NPLTA = Empréstimo não recebido sobre Ativo Total.

$$
\text { NLTA }=\frac{\text { Crédito Líquido }}{\text { Ativo Total }}
$$

Que destaca o NLTA = Crédito Líquido sobre Ativo Total.

$$
\text { PXTOX }=\frac{\text { Despesa com Pessoal }}{\text { Despesa Operacional Total }}
$$

Que se utiliza o PXTOX = Despesa com Pessoal sobre Despesa Operacional Total.

$$
\text { OVTA }=\frac{\text { Despesa Geral }}{\text { Ativo Total }}
$$

Em que o OVTA = Despesa Geral sobre Ativo Total.

$$
\text { TOITA }=\frac{\text { Receita Total }}{\text { Ativo Total }}
$$

Que destaca o TOITA = Receita Total sobre Ativo Total.

$$
\mathrm{ROE}=\frac{\text { Sobra Líquida }}{\text { Patrimônio Líquido }}
$$

Para o ROE = Sobra Líquida sobre Patrimônio Líquido.

$$
\text { ROA }=\frac{\text { Sobra Líquida }}{\text { Ativo Total }}
$$

Que se utiliza o ROA = Sobra Líquida sobre Ativo total.

$$
\text { PCDL }=\frac{\text { Provisão para Crédito de Liquidação Duvidosa }}{\text { Ativo Total }}
$$

Onde o PCDL = Provisão para Crédito de Liquidação Duvidosa sobre Atito Total.

$$
\text { TDTA }=\frac{\text { Depósito Total }}{\text { Ativo Total }}
$$

Em que o TDTA = Depósito Total sobre Ativo Total.

$$
\text { LTC }=\frac{\text { Depósito Total }}{\text { Patrimônio Líquido }}
$$

Que se utiliza o LTC = Depósito Total sobre Patrimônio Líquido.

Pesquisas que utilizaram esses indicadores mencionados destacam sua contribuição para a mensuração do desempenho econômico-financeiro, conforme Meyer e Pifer (1970), Sinkey Jr. (1975), Martin (1977), Parliament, Lennan e Fulton (1990), Cornett e Tehranian (1992), Kumar e Arora (1995), Yeh (1996) e Godlewsk (2003). Dos 12 indicadores de desempenho econômico-financeiro utilizado, houve possibilidade de pelo menos dois de cada grupo, o que permitiu a utilização da nomenclatura CAMEL, como apresentada na análise dos resultados da pesquisa.

Foi estabelecido o crescimento da população economicamente ativa, considerando os eleitores de cada município, pela área de atuação da cooperativa. Esses dados foram obtidos no sítio do Tribunal Superior Eleitoral (2011). Considerando que o censo estatístico brasileiro produzido pelo Instituto Brasileiro de Geografia e Estatística (IBGE) ocorre com intervalos de dez anos, foi utilizado o intervalo publicado pelo TSE, que é anual. Destaca-se que o primeiro ano utilizado foi o de 2000, depois 2002, e posteriormente os eleitores de cada ano.

Foram considerados como população economicamente ativa os eleitores aptos a votar, que compreendem a faixa-etária de 16 a 70 anos, configurando população idêntica ao 
estabelecido pelo IBGE, não causando, assim, distorções no entendimento conceitual para esta pesquisa.

Para verificação do grau e do limite de crescimento da população, foi seguido o que destacou Verhulst (1845) que, preocupado em entender a dinâmica dos sistemas ecológicos, formulou sua lei sobre o crescimento de populações, levando em consideração o seu limite. Com efeito, Verhulst modelou algebricamente o crescimento populacional em duas frentes (KRABS; PICKL, 2007). Primeiramente Verhulst supôs o crescimento demográfico de uma espécie em um território, no qual o habitante encontra facilidades (alimentos) de sobrevivência. Chamando de $\lambda$ a taxa de crescimento da espécie Verhulst propôs:

$$
\kappa=\frac{P_{t+1}-P_{t}}{P_{t}}
$$

(Equação 13)

Verhulst confrontou essas duas igualdades: $\frac{P_{t+1}-P_{t}}{P_{t}}=\lambda\left(1-P_{t}\right)$, chegando a sua formulação final:

Em que:

$$
P_{t+1}=P_{t}(1+\lambda)-\lambda P_{t}^{2}
$$

$P$ : número de indivíduos,

$t+1$ : número do período atual adicionado de um (próximo período),

$t:$ número do período atual,

$\lambda$ : taxa de crescimento da população

1 : população economicamente ativa

Com esse modelo, Verhulst pode variar o parâmetro $\lambda$, simulando diversos tipos de ecossistemas, para uma dada quantidade inicial de indivíduos, limitado a uma população $P_{\text {lim. }}$. (VERHULST, 1845). O sistema dinâmico descrito também foi utilizado nas pesquisas de Pearl e Reed (1920), Wolfe (1927), Modis (1999) e Sprott, Wildenberg e Azizi (2005).
Para validar o nível de risco positivo, foram utilizadas as equações de regressões simples "com única variável dependente para cada valor da variável independente" (HAIR JR., 2009, p. 153). Conforme destacam Corrar, Theóphilo e Bergmann (2004), o modelo de regressão linear bem como a equação de regressão linear ajustada aos dados podem ser apresentados como:

Com:

$$
\hat{Y}=\hat{a} x+\hat{b}+\varepsilon
$$

(Equação 15)

$\widehat{Y}$ : a estimativa da variável dependente

e as estimativas dos parâmetros do modelo;

$\hat{a}$ : coeficiente linear;

$\hat{b}$ : coeficiente angular

e a variável explicativa (independente);

$\mathrm{x}$ : valores amostrais da variável independente;

$\varepsilon:$ o erro da regressão.

Conforme representações dos dados coletados, com os dados históricos dos dez anos, ajustou-se o modelo de regressão úni- ca simples, tanto para o Patrimônio Líquido (PL), como para o Ativo Total (AT) e o número de sócios, os modelos ajustados foram:

$$
P L=\alpha \text { sócios }+\beta+\varepsilon
$$

A equação 16 foi utilizada para a projeção do patrimônio líquido. 
$A T=\gamma$ Sócios $+\delta+\varepsilon$

(Equação 17)

A equação 17 foi utilizada para a projeção do ativo total.
Posteriormente, se utilizou da regressão linear múltipla Cunha e Coelho (2007), em continuidade aos procedimentos do modelo linear simples, em que:

Em que:

$$
Y=\beta_{0}+\beta_{1} X_{1}+\beta_{2} X_{2}+\ldots+\beta_{k} X_{k}+\varepsilon
$$

$Y$ : é a variável dependente;

$\mathrm{X}_{1}, \mathrm{X}_{2} \ldots \mathrm{X}_{\mathrm{k}}$ : são variáveis independentes (explicativas);

$\beta_{0}$ : constante da regressão;

$\beta_{1}, \beta_{2} \ldots \beta_{\mathrm{k}}$ : são os parâmetros da regressão;

$\varepsilon$ : erro da regressão ou componente estocástico do modelo.

A projeção dos sócios foi mensurada de acordo com a equação de regressão múltipla "com duas ou mais variáveis independentes" (HAIR JR., 2009, p. 153), que representa a equação de regressão para a projeção do primeiro indicador de capital, do patrimônio líquido sobre o ativo total. Foi estabelecida a equação para o indicador de desempenho, do modelo CAMEL, EQTA, que utiliza o patrimônio líquido sobre o ativo total, como segue:

$$
E Q T A=\alpha_{1} \text { Sócios }+\alpha_{0}+\varepsilon
$$

Posteriormente, foi constituída a equação de regressão para a projeção dos sócios, como segue:

$$
\text { Sócios }=\beta_{0}+\beta_{1} L n P L+\beta_{2} \operatorname{Ln} A T+\varepsilon
$$

Em seguida, foi estabelecida a equação de regressão para a projeção do EQTA, com a substituição dos Sócios na equação 20, na equação 19, como segue:

$$
E Q T A=\alpha_{1}\left(\beta_{0}+\beta_{1} \operatorname{LnPL}+\beta_{2} \operatorname{LnAT}\right)+\alpha_{0}+\varepsilon
$$

Que se considera:

EQTA : Patrimônio Líquido sobre o Ativo Total

LnPL : Logaritmo Neperiano do Patrimônio Líquido

LnAt : Logaritmo Neperiano do Ativo Total

O nível de risco positivo estabelecido pela equação 14 determina o desempenho desejado para a cooperativa de crédito, porém considera-se fundamental que esse nível seja equilibrado para o período estipulado, pois, em caso de resultado indesejado, necessita-se estabelecer novos valores para que a projeção retome o resultado esperado.

Esta pesquisa considerou a premissa de que será mantido, como direcionador do resultado, o princípio da continuidade da entidade, em que, conforme Hendriksen e Van Breda (1999), o resultado desejado está alinhado com a continuidade da atividade no atendimento às necessidades de uma sociedade.

Com base nas áreas de atuação, representadas pelos municípios em que as cooperativas possuem os PAC's, foi possível efetivar a busca pela população economicamente ativa de cada município. Esses dados foram coletados no sítio do Supremo Tribunal Eleitoral (STE), considerada a população com possibilidade de voto e as obrigadas ao voto, que equivalem à população economicamente ativa de cada região de abrangência das cooperativas.

A análise dos dados aconteceu depois de calculados os indicadores do modelo CAMEL, 
descrito por Meyer e Pifer (1970), representados em cinco grupos. Foram analisados os resultados com alterações representativas, em cada indicador e em cada grupo de indicadores, de um período para outro de 2001 até 2010, de forma transversal e longitudinal, conforme o indicado por Gitman (2004), com representação no desempenho econômico-financeiro de cada cooperativa, de forma longitudinal, além da comparabilidade do comportamento no mercado, de forma transversal.

Em seguida, foram calculadas as regressões lineares simples para determinação da quantidade de população e dos cooperados para o período futuro de 15 anos. Foi estabelecido o período de projeção com base na necessidade do modelo de crescimento populacional, que se caracteriza como projeção de longo prazo. Esse resultado permitiu a análise de como seria o crescimento das cooperativas, com base no que vem ocorrendo nos últimos períodos estudados.

Posteriormente, foi utilizado o modelo de Verhulst, considerando o período estipulado de projeção de 15 anos, que permitiu obter o limite de crescimento da população, em que a cooperativa poderia atingir como sócios. O limite permitiu saber o ano de projeção em que a cooperativa conseguiria ter toda a população economicamente ativa como sócia. Para o acréscimo do número de cooperados, nas variáveis da pesquisa, considerou-se a função $Y=f(t, p)+\varepsilon$, em que o crescimento do número de cooperados $Y$ depende das variáveis $t$, para tempo, e $p$, para a população economicamente ativa. Assim, a função seria:

$$
\text { N.Coop }=f(t . p)+\varepsilon
$$

(Equação 21)

Com a determinação do crescimento natural e considerada a população economicamente ativa, como sendo o limite de desempenho das cooperativas na busca por novos sócios, foi estabelecido o nível de risco positivo otimizado, que, descontado do risco natural, representa o intervalo de desenvolvimento possível para cada cooperativa de crédito, considerando que o ambiente se desenvolva na mesma situação apresentada.

Posteriormente, as variáveis econômico-financeiras foram organizadas em forma de tabela, conforme a composição dos indicadores, calculado o Logaritmo Neperiano para normalização dos valores, conforme destacado por Carleton (1970), Grace, Harrington e Klein (1998), McKernan (2002), Bekaert, Harvey e Lundblad (2007) e Sahut e Mili (2011).

Em sequência, para validar o nível de risco positivo na atividade de crédito para otimização do desempenho econômico-financeiro das cooperativas de crédito objeto do estudo, foram estabelecidas equações de regressões lineares simples e múltiplas, por variável, para todos os indicadores. Considerado o grau de risco positivo para o crescimento da população economicamente ativa, foram recalculados os indicadores e comparados o real com o projetado, com base no desenvolvimento da população economicamente ativa.

Em relação às limitações da pesquisa, considerou-se que a utilização dos métodos e modelos escolhidos pode representar algum fato limitante. $\mathrm{O}$ modelo CAMEL não pode ser utilizado na sua plenitude, pois, dos 27 indicadores do modelo apresentado por Daley, Matthews e Whitfield (2008), foi possível a utilizar 12 indicadores, os demais ficaram impossibilitados na coleta de variáveis, pois as demonstrações disponibilizadas não apresentavam o nível de detalhamento necessário à coleta. Considera-se, também, uma limitação desta pesquisa a utilização do modelo de Verhulst; mesmo com sua utilização em pesquisas na área de finanças, ainda não havia sido destacada sua utilização no desempenho de cooperativas de crédito, em que, além do modelo, o ambiente pode ser restritivo.

Ainda, como fator limitante na pesquisa pode ser considerada a amostra. A utilização de dez cooperativas, por dez anos, destaca o desempenho econômico-financeiro dessas cooperativas, porém não representa a maioria delas, mesmo que essas cooperativas utilizadas representem $29,35 \%$ do ativo das 100 maiores cooperativas e, com $16,68 \%$ do total de ativos das 1370 cooperativas de crédito brasileiras.

\section{ANÁLISE DOS DADOS}

Em relação à população economicamente ativa, foi calculado, com base no crescimento atual, o número de pessoas que essa população teria para o período projetado, de 15 anos. Assim, ao estabelecer o número máximo de pessoas de um ambiente, pode-se projetar o crescimento do número de 
cooperados, considerando as áreas de atuação, das cooperativas de crédito.

Os resultados apresentados na tabela 1 destacam o coeficiente não padronizado de 192199,315 para a População/ano, adicionando 10495722,067 como uma constante.
Os testes de significância, para a população e para o período, foram inferiores a $0,05 \%$, o que permite a consideração de relação significante para essas variáveis (MAROCO, 2003). Considera-se a projeção da População para o ano 2025 (15 anos), na tabela 1.

$$
\begin{gathered}
\text { EqPop }=a P e r+b+\varepsilon \\
\text { EqPop }=19219915+1,04957\left(10^{7}\right) \\
\text { Pop }=15.492 .874
\end{gathered}
$$

(Equação 22)

Tabela 1 - Equação de regressão da população para 15 períodos posteriores

\begin{tabular}{l|l|c}
\hline \multicolumn{1}{c|}{ Cooperativa } & Pop_Equação de Regressão & Projeção da População \\
\hline 1- Credicitrus & Pop1 $=192199 *$ Ano $+1,04957 \mathrm{e}+007$ & 15.492 .874 \\
\hline 2- Cooperforte & Pop2 $=230163 *$ Ano $+1,54587 \mathrm{e}+007$ & 21.442 .938 \\
\hline 3- Cocred & Pop3 $=33850,7 *$ ano $+2,03973 \mathrm{e}+006$ & 2.919 .848 \\
\hline 4- Viacredi & Pop4 $=18862,6$ * Ano +622303 & 1.112 .730 \\
\hline 5- Pioneira & Pop5 $=21250,5 *$ Ano +606059 & 1.158 .572 \\
\hline 8- Região dos Vales & Pop8 $=6737,78 *$ Ano +718038 & 893.220 \\
\hline 9- Coopecredi & Pop9 $=19113,9 *$ Ano +799075 & 8.296 .036 \\
\hline 10- Credicoamo & Pop10 $=6737,78 *$ Ano +718038 & 729.149 \\
\hline 11- Cataratas do Iguaçu & Pop11 $=10899,5 *$ Ano +445763 & 2.794 .036 \\
\hline 12- Uniprime & Pop12 $=23831 *$ Ano $+2,17443 e+006$ & \\
\hline
\end{tabular}

Fonte: Dados da pesquisa.

Posteriormente ao estabelecimento do limite de crescimento da população para o período de projeção, com base no crescimento anteriormente coletado, foi apurada a equação de regressão para o crescimento dos cooperados, com a mesma base de períodos, com os mesmos procedimentos.
Os testes de significância também foram inferiores a $0,05 \%$. Foi considerada, também, a projeção da população para o ano 2025 (15 anos), destacados na tabela 2 .

$$
\begin{gathered}
\text { EqCoop }=a \text { Per }+b+\varepsilon \\
E q \text { Coop }=4561,815+(-4157,2) \\
E q \text { Coop }=114.450
\end{gathered}
$$

Tabela 2 - Equação de regressão dos cooperados para 15 períodos posteriores

\begin{tabular}{l|l|c}
\hline \multicolumn{1}{c|}{ Cooperativa } & \multicolumn{1}{c}{ Pop_Equação de Regressão } & Projeção dos Cooperados \\
\hline 1-Credicitrus & Coop $1=4561,8$ * Ano $+(-4157,2)$ & 114.450 \\
\hline 2-Cooperforte & Coop $2=6512,33$ * Ano $+44079,2$ & 213.400 \\
\hline 3-Cocred & Coop $3=1487,79 *$ ano $+(-1583,47)$ & 37.099 \\
\hline 4-Viacredi & Coop $4=14131,5 *$ Ano $+(-21605,3)$ & 845.814 \\
\hline 5-Pioneira & Coop $5=5047,54$ * Ano $+10395,9$ & 141.631 \\
\hline 8-Região dos Vales & Coop $8=1945,3 *$ Ano $+16036,3$ & 3.810 \\
\hline 9-Coopecredi & Coop $9=114,073 *$ Ano $+844,4$ & 33.167 \\
\hline 10-Credicoamo & Coop $10=660,091 *$ Ano $+16004,6$ & 114.636 \\
\hline 11-Cataratas do Iguaçu & Coop $11=4280,23 *$ Ano $+3350,13$ & 22.965 \\
\hline 12-Uniprime & Coop $12=841,176 *$ Ano $+1079,73$ & \\
\hline
\end{tabular}

Fonte: Dados da pesquisa. 
A equação de regressão dos cooperados foi utilizada para o cálculo do limite de cooperados, ao final do período estabelecido, que foi considerado como o crescimento natural da cooperativa, pelo ingresso de cooperados. Foi estabelecido o crescimento dos cooperados em risco natural como Coop_ $m$, pelo crescimento da forma como vem ocorrendo nas cooperativas de crédito, para que, ao final do período, no ano de 2025, estivesse representado o crescimento obtido pela equação de regressão.

O crescimento do número de cooperados ocorre de forma equalizada quando observado o risco positivo otimizado, de forma que o crescimento da cooperativa projeta o crescimento sem comprometer a continuidade da cooperativa, analisado pelos indicadores econômico-financeiros. Esse crescimento, de forma prudente, representa a taxa de risco positivo que eleva a participação na competitividade, porém, com um grau de segurança, dentro do intervalo de risco positivo, que foi estabelecido com reserva antes de atingir o limite da população economicamente ativa no ambiente de atuação da cooperativa. A tabela 3 destaca o risco positivo otimizado por cooperativa.

Tabela 3 - Risco Positivo Otimizado por Cooperativa

\begin{tabular}{l|c|c|c}
\hline \multicolumn{1}{c|}{ Cooperativa } & Risco Natural (\%) & Risco Positivo Otimizado (\%) & RP - RN (\%) \\
\hline 1-Credicitrus & 9,27 & 2,69 & $-6,58$ \\
\hline 2-Coperforte & 7,76 & 8,82 & 0,53 \\
\hline 3-Cocred & 9,74 & 4,57 & $-5,17$ \\
\hline 4-Viacredi & 7,76 & 15,75 & 7,98 \\
\hline 5-Pioneira & 6,81 & 1,12 & $-5,69$ \\
\hline 8-Região dos Vales & 9,08 & 3,51 & $-5,57$ \\
\hline 9-Coopecredi & 6,04 & 1,20 & $-4,84$ \\
\hline 10-Credicoamo & 1,63 & 3,56 & 1,93 \\
\hline 11-Cataratas & 3,44 & 5,17 & 1,74 \\
\hline 12-Uniprime & 13,63 & 2,99 & $-10,64$ \\
\hline
\end{tabular}

Fonte: Dados da pesquisa.

O risco positivo limite destaca o quanto a cooperativa pode aumentar o volume de cooperados, sem observar os indicadores econômico-financeiros, com um grau de conservadorismo em que, anteriormente ao total da população economicamente ativa projetada, se consiga o desenvolvimento da cooperativa na atividade.

O limite do risco positivo otimizado representa o percentual de crescimento para o número de cooperados, em situação que a cooperativa mantenha relativa segurança no aumento desses cooperados. E existe a possibilidade de captação de cooperados, no entanto, se deve observar um intervalo que represente a prudência para que as cooperativas possam determinar ações de crescimento, com manutenção de um grau que represente segurança em relação à população economicamente ativa, na sua área de atuação.

O risco positivo otimizado representa um grau de risco que permite a verificação do desempenho econômico-financeiro, de forma que a cooperativa apresente todos os indica- dores positivos. No entanto, caso os gestores das cooperativas optem pela exposição de algum indicador negativo, podem elevar o grau de risco positivo de forma que atenda as necessidades desejadas no desempenho econômico-financeiro.

Após a análise do grau de risco positivo otimizado, com a verificação do desempenho econômico-financeiro de cooperativas de crédito, foram inseridos os indicadores do modelo CAMEL. No estabelecimento do limite do risco positivo otimizado, foi estabelecido que todos os indicadores apresentassem resultado positivo, no entanto poderia apresentar um desempenho conservador que represente uma diferença próxima ao desempenho pelo risco natural, sem que os resultados entrem em ambiente turbulento em todos os indicadores.

Com a simulação do intervalo de risco positivo às atividades de crédito para o crescimento de cooperativas de crédito, que separa o risco natural do limite do risco positivo, configura-se o intervalo de risco possível. Como 
intervalo de risco definido foram simuladas taxas que permitissem descobrir a posição, no intervalo e atingir o desempenho econômico-financeiro pretendido no período estipulado. Posteriormente, ocorre a validação do nível de risco positivo otimizado com o desempenho econômico-financeiro na atividade de crédito de cooperativas de crédito.

\subsection{Risco positivo otimizado no desempenho econômico-financeiro}

A análise utiliza a continuação dos indicadores analisados anteriormente, que, otimizados pelo nível de risco positivo, permitiram a verificação do desempenho nos indicadores, fato que foi considerado adequado quando todos os indicadores foram equilibrados com resultado positivo projetado para os próximos 15 (quinze) anos, em que este refinamento precisa ser ajustado a cada período que passa alinhado com ações estratégicas das cooperativas de crédito brasileiras.

Retomando as indicações, será apresentado o risco natural, pela situação como vinha sendo obtido pelo desempenho econômico-financeiro analisado anteriormente, com a adequação ao desempenho direcionado pelo risco positivo na projeção dos indicadores.

A tabela 4 apresenta o indicador do patrimônio líquido sobre o ativo total sob risco positivo para a cooperativa Credicitrus, em que, com a utilização do grau de risco positivo para o aumento dos sócios, foi projetado o desempenho econômico-financeiro do patrimônio líquido e do ativo total.

Tabela 4 - Indicador do patrimônio líquido sobre o ativo total sob risco positivo - Credicitrus

\begin{tabular}{c|c|c|c|c|c|c|c}
\hline Ano & Sócios & PL & AT & EQTA & LnPL & LnAT & EQTArp \\
\hline 2011 & 44.382 & 560.752 .603 & 2.124 .525 .920 & 0,263942 & 20,14479 & 21,477 & 0,260 \\
\hline 2012 & 45.573 & 576.840 .155 & 2.182 .935 .181 & 0,26425 & 20,17308 & 21,504 & 0,260 \\
\hline 2013 & 46.795 & 593.346 .442 & 2.242 .864 .749 & 0,264548 & 20,20129 & 21,531 & 0,260 \\
\hline 2014 & 48.050 & 610.298 .480 & 2.304 .412 .710 & 0,264839 & 20,22946 & 21,558 & 0,261 \\
\hline 2015 & 49.338 & 627.696 .269 & 2.367 .579 .064 & 0,265122 & 20,25757 & 21,585 & 0,261 \\
\hline 2016 & 50.661 & 645.566 .824 & 2.432 .461 .894 & 0,265396 & 20,28564 & 21,612 & 0,262 \\
\hline 2017 & 52.019 & 663.910 .144 & 2.499 .061 .202 & 0,265664 & 20,31366 & 21,639 & 0,262 \\
\hline 2018 & 53.414 & 682.753 .246 & 2.567 .475 .071 & 0,265924 & 20,34164 & 21,666 & 0,262 \\
\hline 2019 & 54.846 & 702.096 .130 & 2.637 .703 .501 & 0,266177 & 20,36958 & 21,693 & 0,263 \\
\hline 2020 & 56.316 & 721.952 .302 & 2.709 .795 .535 & 0,266423 & 20,39747 & 21,720 & 0,263 \\
\hline 2021 & 57.825 & 742.335 .270 & 2.783 .800 .215 & 0,266663 & 20,42531 & 21,747 & 0,263 \\
\hline 2022 & 59.375 & 763.272 .050 & 2.859 .815 .625 & 0,266896 & 20,45313 & 21,774 & 0,264 \\
\hline 2023 & 60.965 & 784.749 .134 & 2.937 .792 .723 & 0,267122 & 20,48087 & 21,801 & 0,264 \\
\hline 2024 & 62.599 & 806.820 .552 & 3.017 .927 .678 & 0,267343 & 20,50861 & 21,828 & 0,265 \\
\hline 2025 & 64.276 & 829.472 .798 & 3.100 .171 .447 & 0,267557 & 20,5363 & 21,855 & 0,265 \\
\hline
\end{tabular}

Fonte: Dados da pesquisa

Onde:

PL = Patrimônio Líquido;

$\mathrm{AT}=$ Ativo Total;

EQTA = Patrimônio Líquido sobre Ativo Total;

LnPL = Logaritmo Neperiano do Patrimônio Líquido;

LnAT $=$ Logaritmo Neperiano do Ativo Total;

EQTA rp = Patrimônio Líquido sobre Ativo Total sob Risco Positivo;

Pela equação de regressão 16, 17 e 19, respectivamente $P L=-3,87417$ e+007 +13507,6. Sócios e AT $=-5,2065 \mathrm{e}+007+49042,2$ Sócios e EQTA = 0,224839 + 8,81853e-007Sócios, utilizadas para cálculo da projeção do resultado a partir do ano de 2011. 
Em relação à projeção do indicador EQTA sob risco positivo, foi utilizada a equação de regressão múltipla 18, dos Sócios $=-245341+28728,8 \mathrm{LnPL}-13686,9 \mathrm{LnAT}$, que foi consolidada com a 19, EQTA $=0,224839+$ 8,81853e-007Sócios, o que permitiu a projeção dos índices sob risco positivo, apresentada para a Credicitrus, em 2011, de 0,260 e finaliza, em 2025, com 0,265.
Este primeiro indicador destaca a utilização do patrimônio líquido sobre o ativo total, na tabela 5, que representa o aumento de capital equiparado com o aumento do ativo, que destaca um direcionador do desempenho econômico-financeiro, em que o aumento de sócios contribui para o aumento de capital e o aumento do ativo, além do aumento que resultou do gerenciamento da instituição de crédito.

Tabela 5 - Indicador do patrimônio líquido sobre o ativo total sob risco positivo

\begin{tabular}{l|l|l|l|l|l|l|l|l|l|l|l|l|l|l|l}
\hline EQTA & $\mathbf{2 0 1 1}$ & $\mathbf{2 0 1 2}$ & $\mathbf{2 0 1 3}$ & $\mathbf{2 0 1 4}$ & $\mathbf{2 0 1 5}$ & $\mathbf{2 0 1 6}$ & $\mathbf{2 0 1 7}$ & $\mathbf{2 0 1 8}$ & $\mathbf{2 0 1 9}$ & $\mathbf{2 0 2 0}$ & $\mathbf{2 0 2 1}$ & $\mathbf{2 0 2 2}$ & $\mathbf{2 0 2 3}$ & $\mathbf{2 0 2 4}$ & $\mathbf{2 0 2 5}$ \\
\hline 1-Credicitrus & 0,260 & 0,260 & 0,260 & 0,261 & 0,261 & 0,262 & 0,262 & 0,262 & 0,263 & 0,263 & 0,263 & 0,264 & 0,264 & 0,265 & 0,265 \\
\hline 2-Coperforte & 0,228 & 0,221 & 0,214 & 0,207 & 0,200 & 0,193 & 0,187 & 0,180 & 0,174 & 0,168 & 0,162 & 0,155 & 0,149 & 0,143 & 0,137 \\
\hline 3-Cocred & 0,119 & 0,118 & 0,117 & 0,116 & 0,115 & 0,114 & 0,113 & 0,112 & 0,111 & 0,110 & 0,109 & 0,108 & 0,107 & 0,105 & 0,104 \\
\hline 4-Viacredi & 0,200 & 0,194 & 0,190 & 0,185 & 0,181 & 0,178 & 0,175 & 0,173 & 0,170 & 0,169 & 0,167 & 0,165 & 0,164 & 0,163 & 0,162 \\
\hline 5-Pioneira & 0,187 & 0,187 & 0,188 & 0,188 & 0,189 & 0,189 & 0,189 & 0,190 & 0,190 & 0,191 & 0,191 & 0,192 & 0,192 & 0,192 & 0,193 \\
\hline 8-Região Vales & 0,150 & 0,152 & 0,153 & 0,155 & 0,156 & 0,158 & 0,159 & 0,160 & 0,161 & 0,162 & 0,163 & 0,164 & 0,165 & 0,166 & 0,167 \\
\hline 9-Coopecredi & 0,039 & 0,037 & 0,035 & 0,033 & 0,031 & 0,029 & 0,027 & 0,025 & 0,023 & 0,021 & 0,019 & 0,018 & 0,016 & 0,014 & 0,012 \\
\hline 10-Credicoamo & 0,554 & 0,560 & 0,565 & 0,571 & 0,576 & 0,581 & 0,585 & 0,590 & 0,594 & 0,598 & 0,603 & 0,607 & 0,610 & 0,614 & 0,618 \\
\hline 11-Ctas_Iguaçú & 0,150 & 0,149 & 0,148 & 0,146 & 0,145 & 0,144 & 0,142 & 0,141 & 0,140 & 0,138 & 0,137 & 0,136 & 0,135 & 0,133 & 0,132 \\
\hline 12-Uniprime & 0,208 & 0,210 & 0,212 & 0,214 & 0,216 & 0,218 & 0,220 & 0,222 & 0,224 & 0,227 & 0,229 & 0,231 & 0,233 & 0,235 & 0,237 \\
\hline
\end{tabular}

Fonte: Dados da pesquisa.

Para os índices da tabela 5, destaca-se a projeção de resultado, sob risco positivo otimizado da cooperativa Credicitrus, que destaca uma elevação continuada, do ano de 2011, com índice de 0,260, até ano de 2025, com índice de 0,265. Essa variação elevada, mas próxima, foi o resultado do refinamento do risco positivo que apresentasse todos os indicadores do modelo CAMEL utilizados nesta pesquisa, com resultado positivo, fato que, se os gestores aceitarem algum indicador com resultado positivo, poderá levar a outros indicadores que representem o desempenho econômico-financeiro com aumento além do que o apresentado nesta fase da análise. Assim, o refinamento do grau de risco positivo pode ser maior, no entanto o objeto desta pesquisa foi de manter os indicadores com resultado positivo, sem turbulência.

A Coopecredi apresenta a menor projeção neste indicador, pois seus resultados apresentam, para o ano de 2025, um índice de 0,0237 , que representa $2,37 \%$. Já a Coperforte e a Viacredi apresentam diminuição dos índices, porém com relativa descida, e, de forma estabilizada, no final do período de projeção.

As demais cooperativas apresentam índices bastante próximos entre elas. A Cocred e a Cataratas do Iguaçu apresentam linha inclinada em direção à redução, porém a inclinação é bastante reduzida. Isso destaca a possibilidade de estabelecimento de ações que proporcionem aumento no desempenho econômico-financeiro e elevação na projeção dos resultados.

Esse indicador apresentou, no período de 2001 até 2010, fortes turbulências que apresentaram alterações, conforme destacado no gráfico 1, que destaca o risco desse indicador em situação de risco natural e em risco positivo, que contribui com a visualização temporal e transversal deste indicador de todas as cooperativas, pelos períodos analisados. 


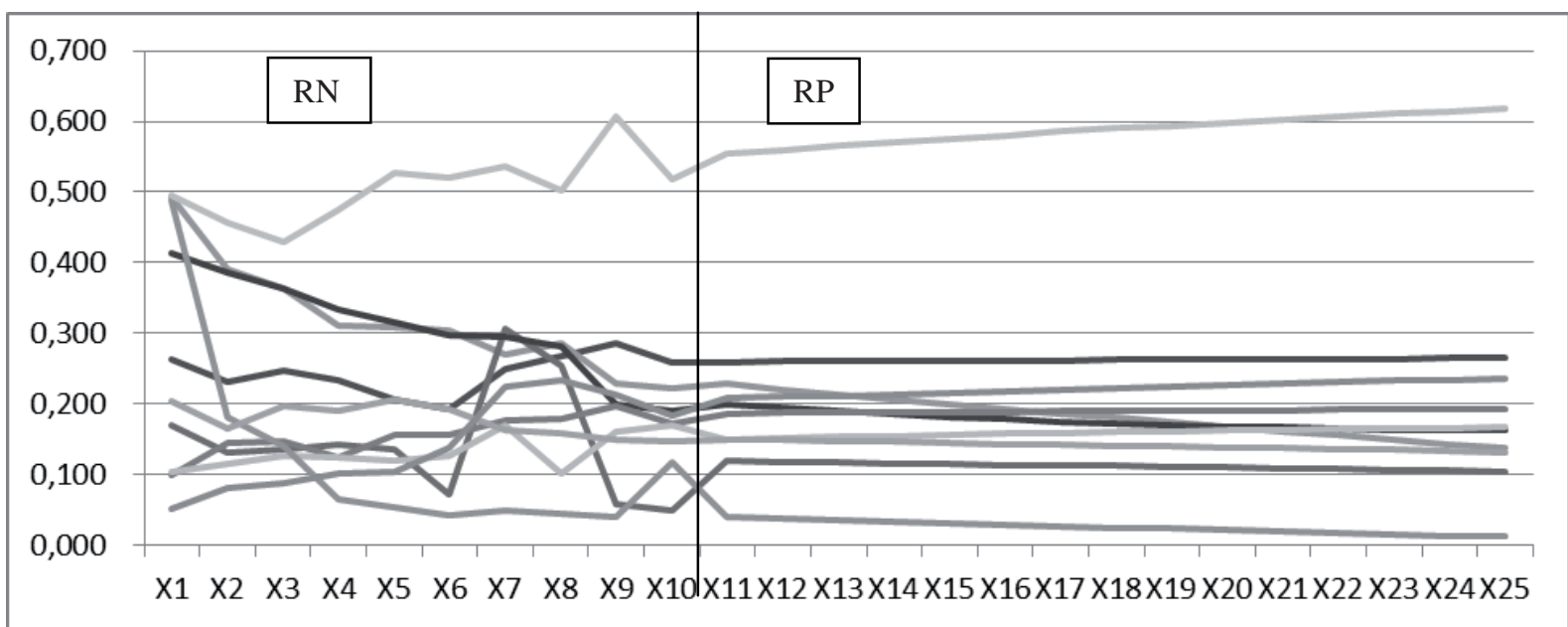

Gráfico 1 - Indicador de patrimônio líquido sobre o ativo total sob risco natural e positivo Fonte: Dados da pesquisa.

As turbulências apresentadas, no caso da Coopecredi, Coperforte, Cocred e Credicitrus destacam que a atuação do risco positivo otimizado permitiu a projeção equalizada do desempenho econômico-financeiro para todas as cooperativas de créditos analisadas.

As projeções estabelecidas pelo risco positivo para o desempenho da atividade de crédito nas cooperativas de crédito compreendem o aporte de recursos dos sócios cooperados em função do aumento de capital que proporciona à cooperativa, no momento em que adere ao cooperativismo de crédito. Essa relação direta destaca o desempenho econômico-financeiro causado na atividade, em que pode ser considerado o nível de desempenho pretendido pelos gestores.

E, considerando a validação do nível de risco positivo na atividade de crédito para otimização do desempenho econômico-financeiro de cooperativas de crédito, que considera o desempenho econômico financeiro equilibrado, os resultados apresentados que destacam o indicador do patrimônio líquido sobre o ativo total sob risco positivo, até o patrimônio líquido sobre o ativo total sob risco positivo, das médias do desempenho econômico-financeiro de 2001 até 2025, foi considerado o desempenho equilibrado entre os grupos de indicadores, para a validação do nível de risco positivo na atividade de crédito.

O risco positivo otimizado no desempenho econômico-financeiro apresenta impactos diferentes para cada cooperativa, sem obede- cer a uma normalidade ou sequência de relação, para a amostra utilizada nesta pesquisa, que configura a validação do risco positivo, com a necessidade de acompanhamento permanente, considerando também, a necessidade de ajuste do nível de risco positivo, a cada novo período projetado, à medida que vão se realizando, o que consiste no refinamento em busca do resultado desejado.

\section{CONCLUSÃO E RECOMENDAÇÕES}

A mensuração do impacto no desempenho econômico-financeiro, com base no estabelecimento do grau de risco positivo otimizado determina patamares na gestão de risco com que as cooperativas de crédito podem estabelecer seu desempenho, sem que entrem em ambiente que resulte em situação de turbulência, de forte oscilação, no seu resultado.

Esse nível de risco positivo define, descontado o risco natural, o ambiente de gestão de risco nas cooperativas, sob a condição de predisposição à folga, anteriormente ao limite possível, considerado que o desempenho econômico-financeiro precisa ocorrer de forma equilibrada no período de planejamento, com a observância no estabelecimento das políticas de crédito e de desempenho das cooperativas de crédito.

O desempenho econômico-financeiro natural, obtido pelos indicadores econômico-financeiros, considerando o desempenho natural, pode resultar em desempenho na 
atividade de crédito. Contudo a validação do nível de risco positivo na atividade de crédito permite a otimização do desempenho econômico-financeiro, em que a adição do grau de risco positivo otimizado, considerado como nível desejado, estabelecerá um o desempenho equilibrado, confirmando a performance econômico-financeira, estabelecido para cada cooperativa, o que representará o desempenho econômico-financeiro otimizado.

Na estruturação do problema, foi estabelecida a questão de pesquisa, que indagava, qual o nível de risco positivo na atividade de crédito que otimiza o desempenho econômico-financeiro de cooperativas de crédito? Com a validação do nível de risco positivo na atividade de crédito para otimização do desempenho econômico-financeiro de cooperativas de crédito, responde-se à questão de pesquisa em que, para cada cooperativa, foi estabelecido o nível de risco positivo que otimiza o desempenho econômico-financeiro de cooperativas de crédito.

Diante dos resultados da pesquisa anteriormente expostos, foi identificado o nível de risco positivo na atividade de crédito que otimiza o desempenho econômico-financeiro de cooperativas de crédito, com destaque para a mensuração do desempenho econômico-financeiro das cooperativas de crédito Meyer e Pifer (1970) e Sinkey Jr. (1975) pelo modelo CAMEL.

O risco positivo otimizado permitiu a mensuração do desempenho econômico-financeiro da atividade na cooperativa de crédito. Foi constatado que o nível do risco positivo equalizado otimiza o desempenho economicamente sustentável da atividade de crédito no desempenho econômico-financeiro das cooperativas de crédito, modus in rebus, para as atividades de crédito.

Como as recomendações, o ambiente de cooperativas de crédito é vasto para o desenvolvimento de pesquisas. As recomendações concentram-se na aplicação de outras metodologias para avaliação do desempenho econômico-financeiro, dando continuidade ao ambiente, como o modelo PEARLS (Protection, Effective financial structure, Asset quality, Rates of Return and Costs, Liquidity, Signs of growth), e conjuntamente o modelo de Verhulst, em contraponto ao modelo CAMEL.

\section{REFERÊNCIAS}

AHARONY, Joseph; JONES, Charles P.; SWARY, Itzhak. An analysis of risk and return characteristics of corporate bankruptcy using capital market data. The Journal of Finance, v. 35, n. 4, p. 1001-1016, set. 1980.

ANDERSEN, Torben Juul. Multinational risk and performance outcomes: effects of knowledge intensity and industry context. International Business Review, p. 1-14, fev. 2011.

ALTMAN, Edward I. Financial ratios, discriminant analysis and the prediction of corporate bankruptcy. The Journal of Finance, v. 23, n. 4, p. 589-609, set. 1968.

Corporate bankruptcy potential, stockholder returns and share valuation. The Journal of Finance, v. 24, n. 5, p. 887-900, dez. 1969.

Railroad bankruptcy propensity. The Journal of Finance, v. 26, n. 2, p. 333-345, maio 1971.

AVERY, Robert B.; BELTON, Terrence M.; GOLBERG, Michael A. Market discipline in regulating bank risk: new evidence from the capital markets. Journal of Money, Credit and Banking, v. 20, n. 4, p. 597-610, nov. 1988.

AVIO, Kenneth L. On the effects of statutory interest rate ceilings. The Journal of Finance, v. 29, n. 5, p. 13831395, dez. 1974.

BANCO CENTRAL DO BRASIL (BACEN). Relatório de gestão. 2010. Disponível em: <www.bcb.gov.br>. Acesso em: 8 dez. 2011.

. Relatório de inclusão financeira. Brasília, DF, n. 2, 2011. Disponível em: <www.bcb.gov.br>. Acesso em: 18 dez. 2011.

BARGER, Harold. Speculation and the risk-preference. The Journal of Political Economy, v. 46, n. 3, p. 396-408, jun. 1938.

BEAVER. William H. Financial ratios as predictors of failure. Journal of Accounting Research, v. 4, p. 71-111, 1966.

BEKAERT, Geert; HARVEY, Campbell R.; LUNBLAND, Christian. Liquidity and expected returns: lessons from emerging markets. The Review of Financial Studies, v. 20, n. 6, p. 1783-1831, nov. 2007.

CARLETON, Willard T. An Analytical Model for LongRange Financial Planning. The Journal of Finance, v. 25, n. 2, p. 291-315, maio 1970.

COOLEY, Philip L. A multidimensional analysis of institutional investor perception of risk. The Journal of Finance, v. 32, n. 1, p. 67-78, mar. 1977.

CORNETT, Marcia Millon; TEHRANIAN, Hassan. Changes in corporate performance associated with bank acquisitions. Journal of Financial Economics, v. 31, p. 211-234, ago. 1992.

CORRAR, Luiz João; THEÓPHILO, Carlos Renato; BERGMANN, Daniel Reed. Regressões. In: CORRAR, Luiz João; THEÓPHILO, Carlos Renato (Org.). Pesquisa operacional para decisão em contabilidade e administração: contabilometria. São Paulo: Atlas, 2004.

CRESWELL, John W. Projeto de pesquisa: métodos qualitativo, quantitativo e misto. 3. ed. Porto Alegre, RS: Artmed-Bookman, 2010. 
CUNHA, Jacqueline Veneroso Alves da; COELHO, Antonio Carlos. Regressão Linear Múltipla. In: CORRAR, Luiz João; PAULO, Edilson; DIAS FILHO, José Maria. Análise multivariada: para cursos de administração, ciências contábeis e economia. São Paulo: Atlas, 2007.

DALEY, J.; MATTHEWS, K.; WHITFIELD, K. Too-bigto-fail: Bank failure and banking policy in Jamaica. Int. Fin. Markets, Inst. and Money, n. 18, p. 290-303, 2008.

DEMIRGÜÇ-KUNT, A.; MAKSIMOVIC, V. Stock market development and financing choices of firms. The World Bank Economic Review, v. 10, p. 341-369, 1996.

DOW, J. C. R. A theoretical account of futures markets. The Review of Economic Studies, v. 7, n. 3, p. 185-195, jun. 1940.

DUFFIE, Darrell; SINGLETON, Kenneth J. Credit risk: princing, measurement, and management. New Jersey: Princeton, 2003.

DZEAWUNI, Wirnkar A.; TANKO Muhammad. Camel(s) and banks performance evaluation: the way forward. Jun. 24, 2008. Disponível em: <http:/ / ssrn.com/ abstract $=1150968>$. Acesso em: jul. 2011.

GIL, Antônio Carlos. Métodos e técnicas de pesquisa social. 5. ed. São Paulo: Atlas, 1999.

GITMAN, Lawrence J. Princípios de administração financeira. 10. ed. São Paulo: Pearson Education do Brasil, 2004.

GODLEWSKI, Christophe J. Modélisation de la prévision de défaillance bancaire et factuers réglementaires: une application aux banques des pays emergents. EconWPA, Finance 0409026, Julliet 2003. Disponível em: <http:/ /econwpa.repec.org/eps/fin/ papers/0409/0409027.pdf>.

GRACE, Martin F.; HARRINGTON, Scott E.; KLEIN, Robert W. Risk-based capital and solvency screening in property-liability insurance: hypotheses and empirical tests. The Journal of Risk and Insurance, v. 65, n. 2, p. 213-243, jun. 1998.

GRINBLATT, Mark; TITMAN, Sheridan. Performance measurement without benchmarks: an examination of mutual fund returns. The Journal of Business, v. 66, n. 1, p. 47-68, jan. 1993.

HAHN, F. H. Uncertainty and the cobweb. The Review of Economic Studies, v. 23, n. 1, p. 65-75, 1955.

HAIR JR., Joseph F. Análise multivariada de dados. 6. ed. PortoAlegre, RS: Bookman, 2009.

HELMBERGER, Peter; HOOS, Sidney. Cooperative enterprise and organization theory. Jornal de Economia do Farm, v., n. 2, maio 1962.

HENDRIKSEN, Eldon S.; VAN BREDA, Michael F. Teoria da contabilidade. São Paulo: Atlas, 1999.

HORRIGAN, James O. The determination of longterm credit standing with financial ratios. Journal of Accounting Research, v. 4, p. 44-62, 1966.

HULME, David. Impact Assessment methodologies for microfinance: theory, experience and better practice. World Development, v. 28, n. 1, p. 79-98, 2000.
HUNJAK, Tihomir; JAKOVČEVIĆ, Drago. AHP based model for bank performance evaluation and rating. 6 th ISAHP, Switzerland, p. 2-4, ago. 2001.

KALDOR, Nicholas. Speculation and economic stability. The Review of Economic Studies, v. 7, n. 1, p. 1-27, out. 1939.

KRABS, Werner; PICKL, Stefan. Modelling, analysis and optimization of biosystems. [S.1.]: Springer, 2007.

KUMAR, Sameer; ARORA, Sant. A model for risk classification of banks. Managerial and Decision Economics, v. 16, n. 2, p. 155-165, mar./abr. 1995.

MAROCO, João. Análise estatística com utilização do SPSS. 2. ed. Lisboa: Sílabo, 2003.

MARSHALL, Christopher Lee. Medindo e gerenciando riscos operacionais em instituições financeiras. Rio de Janeiro: Qualitymark, 2002.

MARTIN, D. Early warning of bank failure: a logit regression approach. Journal of Banking and Finance, v. 1, p. 249-276, 1977.

MARTINS, Gilberto de Andrade; THEÓPHILO, Carlos Renato. Metodologia da investigação científica para ciências sociais aplicadas. São Paulo: Atlas, 2007.

MATTAR, F. N. Pesquisa de marketing: metodologia, planejamento. São Paulo: Atlas, 1997.

McKERNAN, Signe-Mary. The Impact of Microcredit Programs on Self-Employment Profits: Do Noncredit Program Aspects Matter? The Review of Economics and Statistics, v. 84, n. 1, p. 93-115, fev. 2002.

MEEHLING, Charles F. Federal credit unions in the United States: an analysis. The Journal of Finance, v. 14, n. 1, p. 93-94, mar. 1959.

MENG, Ming; NIU, Dongxiao. Modeling CO2 emissions from fossil fuel combustion using the logistic equation. Energy, v. 36, p. 3355-3359, 2011.

MEYER, Paul A.; PIFER, Howard W. Prediction of bank failures. The Journal of Finance, v. 25, n. 4, p. 853-868, set. 1970.

MIRANDA, L. C. M.; LIMA, C. A. S. On the logistic modeling and forecasting of evolutionary processes: Application to human population dynamics. Technological Forecasting \& Social Change, v. 77, p. 699711, 2010

MODIS, Theodore. Technological forecasting at the stock market. Technological Forecasting and Social Change, n. 62, p. 173-202, 1999.

MOODY, J. Carroll; FITE, Gilbert C. The credit union movement: origins and development 1850 to 1980. Iowa: Kendall/Hunt, 1971.

PARLIAMENT, Claudia; LENNAN, Zvi; FULTON, Joan. Performance of cooperatives and investor-owned firms in the dairy industry. Journal of Agricultural Cooperation, p. 1-16, 1990.

PEARL, Raymond; REED, Lowell J. On the rate of growth of the population of the united states since 1790 and its mathematical representation. Proceedings of the National Academy of Sciences, v. 6, n. 6, 15 jun. 1920.

PETTWAY, Richard H.; SINKEY JR., Joseph F. Establishing on-site bank examination priorities: an early-warning system using accounting and market 
information. The Journal of Finance, v. 35, n. 1, p. 137 150, mar. 1980.

RENWICK, Fred B. Asset management and investor portfolio behavior: theory and practice. The Journal of Finance, v. 24, n. 2, p. 181-206, maio 1969.

SAHUT, Jean-Michel; MILI, Mehdi. Banking distress in MENA countries and the role of mergers as a strategic policy to resolve distress. Economic Modelling, v. 28, p. 138-146, 2011.

SECRIST, Horace. National bank failures and non-failures: an autopsy and diagnosis. Bloomington, Ind.: Principia Press, 1938.

SINKEY JR., Joseph F. Multivariate statistical analysis of the characteristics of problem banks. The Journal of Finance, v. 30, n. 1, p. 21-36, mar. 1975.

SPROTT, J. C.; WILDENBERG, J. C.; AZIZI, Y. A simple spatiotemporal chaotic Lotka-Volterra model. Chaos, Solitons and Fractals, n. 26, p. 1035-1043, jan. 2005.
STUHR, David P.; WICKLEN, Robert V. Rating the financial condition of banks: a statistical approach to aid bank supervision. Federal Reserve Bank of New York. Monthly Review, set. 1974.

TRIBUNAL SUPERIOR ELEITORAL (TSE), 2011. Disponível em: <www.tre.gov.br>. Acesso em: fev. 2012. VERHULST, P. F. Loid'acroissement de la population. Recherches of Matématiques. Avril, Bruxelles, 1845.

WOLFE, A. B. Is there a biological law of human population growth? The Quarterly Journal of Economics, v. 41, n. 4, p. 557-594, ago. 1927.

WOLKEN, John D.; NAVRATIL, Frank J. The economic impact of the federal credit union usury ceiling. The Journal of Finance, v. 36, n. 5, p. 1157-1168, dez. 1981.

YEH, Quey-Jen.The application of data envelopment analysis in conjunction with financial ratios for bank performance evaluation. The Journal of the Operational Research Society, v. 47, n. 8, p. 980-988, ago. 1996. 